\title{
Reducing the surgical complications of smoking by cotinine testing
}

To the Editor: We read with interest the edition of SAMJ (November 2013) dedicated to smoking, arguably the most significant modifiable cause of death and disease. One of the areas not addressed relates to the impact of smoking in the context of surgery. It is well recognised that smoking increases the risk of overall complications, arrhythmias, thrombotic episodes, pneumonia, infection, wound healing complications and prolonged hospital stays, as well as the need for further surgery. ${ }^{[1-3]}$ As surgeons we are frequently held responsible for these complications, and yet the decision to undertake the procedure at all should often be scrutinised, especially in the elective setting, rather than just the technical execution thereof.

Use of cotinine, a metabolite of nicotine, has been proposed to detect active smokers who claim not to smoke. This group may comprise as many as $34 \%$ of current smokers, which is not surprising considering the deceptive behaviour of people with addictions in general - in other words, up to a third of active smokers may lie about their smoking status. It has been demonstrated that smokers who 
developed wound healing complications had higher levels of cotinine than those who did not when tested peri-operatively. ${ }^{[1-3]}$

A study by Coon $e t$ al. ${ }^{[1]}$ demonstrated that patients who claimed to have quit smoking were particularly likely to be deceitful, and a cost saving strategy may be employed whereby only this group is tested. In their cohort of plastic surgery patients, the test would detect 1 out of 10 'former' smokers, as opposed to 1 in 66 of all patients in a selfreported non-smoking population.

We believe that routine or selective serum or urine cotinine testing will enable us to stratify risk in both elective and reconstructive scenarios. Many procedures will therefore be cancelled, or at least delayed pending smoking cessation, which may have remarkable cost benefits for health systems, especially one as stretched as ours. Theatre time has been reduced in recent years despite increasing demand, and funding has inexplicably been diverted away from specialist surgical services.

The onus is on surgeons to be far stricter when applying absolute and relative contraindications, such as smoking, to elective surgery. Denying a patient a procedure s/he requires may also provide just the incentive needed to stop smoking.

\section{J Potgieter}

\section{G dos Passos}

\section{E Price}

A D Rogers

Division of Plastic, Reconstructive and Maxillofacial Surgery, Groote Schuur Hospital and Faculty of Health Sciences, University of Cape Town, South Africa

rogersadr@gmail.com

\footnotetext{
1. Coon D, Tiffaha S, Christensen J, Bonawitz SC. Plastic surgery and smoking: A prospective analysis of incidence, compliance, and complications. Plast Reconstr Surg 2013;131(2):385-393. [http://dx.doi org/10.1097/PRS.0b013e318277886a]

2. Marin VP, Ptynia KB, Langstein HN, et al. Serum cotinine concentration and wound complication in head and neck reconstruction. Plast Reconstr Surg 2008;121(2):451-457. [http://dx.doi.org/10.1097/01. prs.0000297833.53794.27]

3. Rohrich RJ, Coberly DM, Krueger JK, Brown SA. Planning elective operations on patients who smoke: Survey of North American plastic surgeons. Plast Reconstr Surg 2002;109(1):350-355. [http://dx.doi.
} org/10.1097/00006534-200201000-00057

S Afr Med J 2014;104(3):154-155. DOI:10.7196/SAMJ.7740 